\title{
1q21.1 microduplication in a patient with mental impairment and congenital heart defect
}

\author{
GUOWEN SUN ${ }^{1,2}$, ZHIPING TAN ${ }^{1,3}$, LIANGLIANG FAN ${ }^{3}$, \\ JIAN WANG ${ }^{1,3}$, YIFENG YANG ${ }^{1,3}$ and WEIZHI ZHANG ${ }^{1,3}$ \\ ${ }^{1}$ Department of Cardiothoracic Surgery, The Second Xiangya Hospital, Central South University, Changsha, Hunan 410011; \\ ${ }^{2}$ Department of Cardiothoracic Surgery, Chenzhou No. 1 People's Hospital, Chenzhou, Hunan 423000; \\ ${ }^{3}$ Clinical Center for Gene Diagnosis and Therapy of State Key Laboratory of Medical Genetics, \\ The Second Xiangya Hospital, Central South University, Changsha, Hunan 410011, P.R. China
}

Received October 16, 2014; Accepted June 11, 2015

DOI: $10.3892 / \mathrm{mmr} .2015 .4166$

\begin{abstract}
. 1q21.1 duplication is a rare copy number variant with multiple congenital malformations, including developmental delay, autism spectrum disorder, dysmorphic features and congenital heart anomalies. The present study described a Chinese female patient (age, four years and eight months) with multiple malformations, including congenital heart defect, mental impairment and developmental delay. The parents and the monozygotic twin sister of the patient, however, were physically and psychologically normal. High-resolution genome-wide single nucleotide polymorphism array revealed a 1.6-Mb duplication in chromosome region 1q21.1. This chromosome region contained $H F E 2$, a critical gene involved in hereditary hemochromatosis. However, the parents and monozygotic twin sister of the patient did not carry this genomic lesion. To the best of our knowledge, the present study was the first to report on a 1q21.1 duplication patient in mainland China.
\end{abstract}

\section{Introduction}

1q21.1 duplication syndrome is a rare aberration of chromosome 1. Chromosome region 1q21.1 contains extensive and complex low-copy repeats. Copy number variants (CNVs) in this region are associated with developmental delay, neuropsychiatric abnormalities, dysmorphic features and a variety of congenital malformations $(1,2)$. The characteristics of 1q21.1 deletion [Online Mendelian Inheritance in Man (OMIM) no. 612474] include developmental delay, microcephaly, facial

Correspondence to: Dr Weizhi Zhang, Department of Cardiothoracic Surgery, The Second Xiangya Hospital, Central South University, 139 Renmin Road, Changsha, Hunan 410011, P.R. China E-mail: zhangwzsmart@gmail.com

Key words: 1q21.1 microduplication, congenital heart defect, highresolution single nucleotide polymorphism, developmental delay, mental impairment anomalies, cataract and congenital heart defect (CHD). Various anatomic types of CHD have been found in 1q21.1 deletion syndromic patients (3), including left-sided obstructions as well as conotruncal and septal defects (4-6). On the other hand, 1q21.1 duplication (OMIM no. 612475) usually leads to mental impairment, autism, macrocephaly and dysmorphic features (1). In addition, a 1q21.1 deletion, as well as 1q21.1 duplication, have been found in patients with apparently non-syndromic CHD. An association between Tetralogy of Fallot (TOF) and 1q21.1 duplication or variants in GJA5 gene mapping in 1q21.1 were documented recently $(7,8)$.

Latest developments of single nucleotide polymorphism (SNP) array allow genome-wide screening at a resolution ( $<5 \mathrm{Mb}$ in size) that is undetectable by traditional cytogenetic methods and have facilitated the discovery of a number of novel microdeletion and microduplication syndromes (9). As part of a larger study on the identification of pathogenic copy number variations in children with CHD (10-12), a high-resolution Illumina SNP array was performed on children with CHD and their parents. The present study provided a detailed report on a Chinese female patient (age, four years and eight months) with developmental delay, neuropsychiatric abnormalities and CHD.

\section{Materials and methods}

Ethical approval and patient consent. The Review Board of the Second Xiangya Hospital of the Central South University approved the present study (Changsha, China). Written informed consent was obtained from the parents of the patient for publication of the present study and any accompanying images.

Clinical presentation. In 2011, a female patient (age, four years and eight months) from Central-South China was seen at the Second Xiangya Hospital of Central South University for a heart murmur. The parents of the patient were unrelated and no family history of inherited diseases was present. At birth, the patient presented with lip cyanosis, clubbing and heart defects (tetralogy of fallot, patent duct artery and patent foramen ovale). At the last examination (at the age of four years and eight months), the patient had a height of $75 \mathrm{~cm}$ and a weight of $10 \mathrm{~kg}$. A moderate mental impairment and a decreased ability 
were observed, and her intelligence quotient was 65 compared to 80 at two years of age. The patient did not present with any distinct facial abnormalities (Fig. 1). The monozygotic twin sister of the patient, however, was physically and psychologically normal.

Cytogenetic analysis. Chromosome analysis was performed by conventional G-banding techniques (550-band resolution) using the peripheral blood samples of the patient, the twin sister and the parents. A sample of $2 \mathrm{ml}$ peripheral blood was collected. All samples were subjected to lymphocyte culture according to a standard cytogenetic protocol.

SNP array analysis. Peripheral venous blood was collected from the patient and her family members. Genomic DNA was isolated from peripheral blood leukocytes using a QIAamp DNA Blood Mini kit (Qiagen, Valencia, CA, USA) according to manufacturer's instructions and was adjusted to a final concentration of $100 \mathrm{ng} / \mu 1$. The Human660w-Quad Chip (Illumina Inc., San Diego, CA, USA) and the Illumina BeadScan genotyping system (Illumina Inc.) were employed to obtain the signal intensities of probes (SNP) following the manufacturer's instructions. The BeadStudio 3.3.7 software (Illumina Inc.) was used to analyze the genotypes [human genome build $36.1(\mathrm{Hg} 18)$ for analysis] and evaluate the experimental quality. The call rates (indicators of genotyping fidelity) of the samples were $>99.0 \%$, indicating that the SNP array results were reliable (11).

PCR-amplified short tandem repeat (STR) analysis. STR analysis was performed on the Applied Biosystems 3130 Genetic Analyzer (Applied Biosystems Life Technologies, Foster City, CA, USA) with the AmpFISTR ${ }^{\circledR}$ Identifiler $^{\circledR}$ PCR Amplification kit (cat. no. 4322288; Invitrogen Life Technologies, Carlsbad, CA, USA) according to the manufacturer's instructions. Data were analyzed using Gene Mapper ${ }^{\circledR}$ ID software (Life Technologies, Carlsbad, CA, USA).

\section{Results}

The chromosome analysis of the patient revealed a normal female karyotype, described as 46,XX. The twin sister and parents also had a normal karyotype. STR-PCR analysis confirmed that the patient and her sister are identical twins (Table I). Clinical examination of the patient showed a combination of phenotypes with CHD, developmental delay, mental impairment, neuropsychiatric abnormalities, raising concerns about a chromosomal abnormality of microdeletion/microduplication. To explore the exact genomic lesion of this patient, the SNP array system (Human660w-Quad Chip, Beadstation Scanner and BeadStudio 3.3.7 software) was employed to analyze the whole genome copy number variations. Comparison with the Database of Genomic Variants (http://dgv.tcag.ca/dgv/app/home) showed a de novo $1.6-\mathrm{Mb}$ duplication at chromosome 1q21.1 (chromosome 1:144972830-146608260) (National Center of Biotechnology Information build $37 / \mathrm{Hg} 19)$. This chromosomal region contains $\sim 30$ annotated genes, including $H F E 2$, HYDIN2, RBM8A and CD160 (Fig. 2). The identical twin sister and parents did not carry this genomic lesion.
Table I. STR-polymerase chain reaction analysis results.

\begin{tabular}{lcc}
\hline STR gene locus & Patient & Twin sister \\
\hline D19S433 & $14.2,17.2$ & $14.2,17.2$ \\
D5S818 & 10,11 & 10,11 \\
D21S11 & 29,32 & 29,32 \\
D18S51 & 12,14 & 12,14 \\
D6S1043 & 10,18 & 10,18 \\
D3S1358 & 15,16 & 15,16 \\
D13S317 & 10,10 & 10,10 \\
D7S820 & 8,11 & 8,11 \\
D16S539 & 9,12 & 9,12 \\
CSF1PO & 11,12 & 11,12 \\
Penta D & 9,14 & 9,14 \\
vWA & 14,14 & 14,14 \\
D8S1179 & 12,16 & 12,16 \\
TPOX & 9,10 & 9,10 \\
Penta E & 11,12 & 11,12 \\
TH01 & 7,9 & 7,9 \\
D12S391 & 17,22 & 17,22 \\
D2S1338 & 23,24 & 23,24 \\
FGA & 23,24 & 23,24 \\
\hline
\end{tabular}

Values represent the genotype of the tested allele. STR, short tandem repeat.

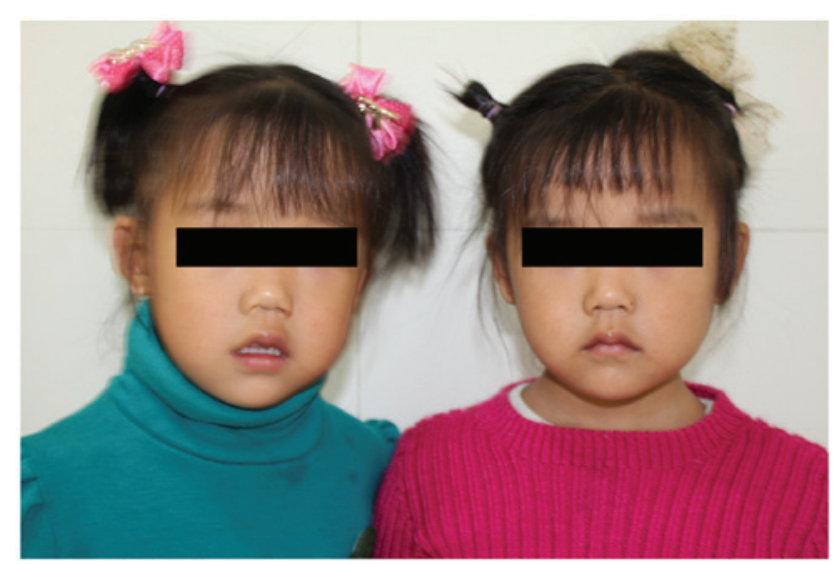

Figure 1. The patient (left, post-operative) and the patient's monozygotic twin sister (right). Neither of them has any distinct facial abnormalities.

\section{Discussion}

The present case report presented a Chinese female patient (age, four years and eight months) with a de novo microduplication of $1.6 \mathrm{Mb}$ in 1q21.1. The patient had a normal karyotype and presented with clinical phenotypes comprising several malformations, developmental delay, neuropsychiatric abnormalities, CHD (tetralogy of fallot, patent duct artery and patent foramen ovale), a moderate mental impairment and a decreased ability. To date, no evidence has suggested that children with cyanotic congenital heart disease suffer from decreased mental performance; therefore, the patient's mental impairment was unlikely 


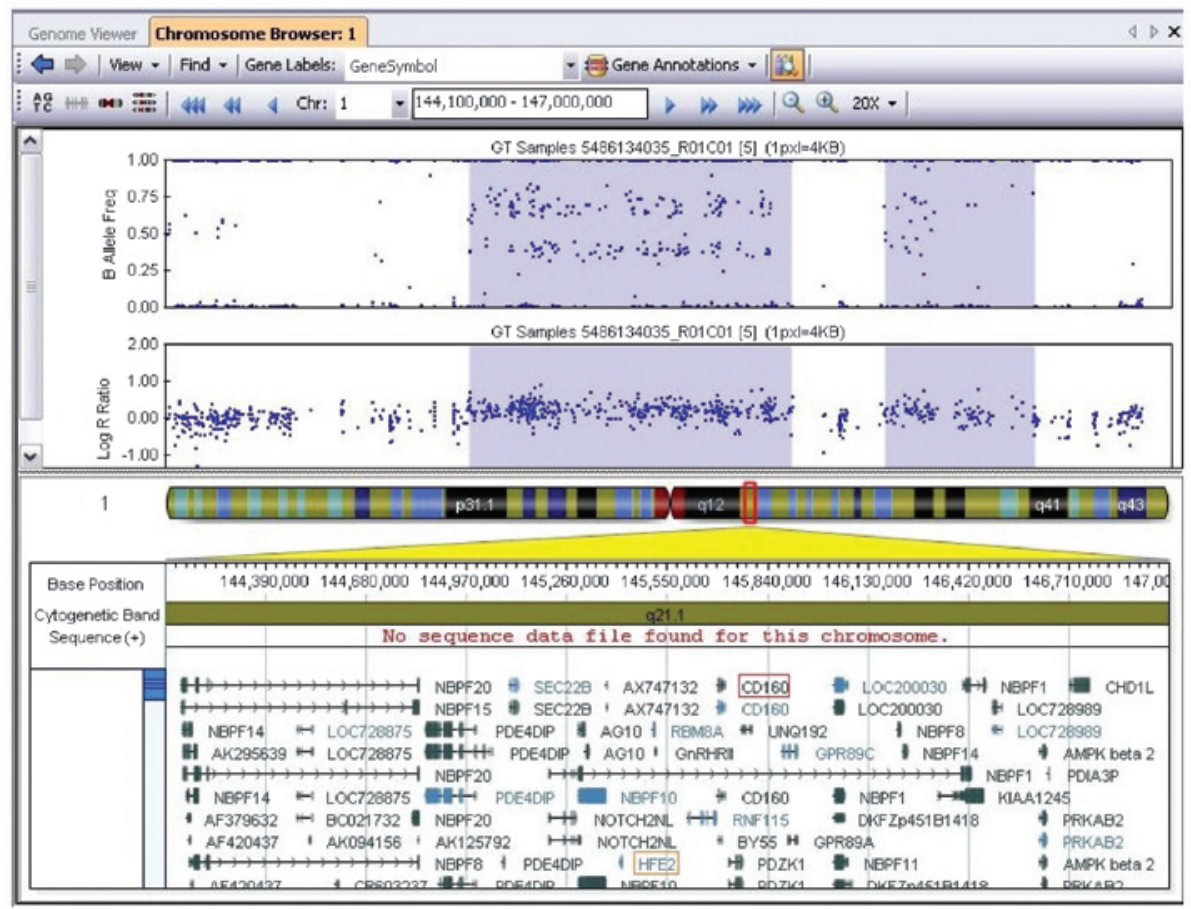

Figure 2. Human 660w-Quad SNP array results of 1q21.1 duplication in the proband. The above panel shows the region involved in cytogenetic bands 1q21.1. A de novo 1.6-Mb duplication on chromosome 1q21.1 (chromosome 1:144972830-146608260) was identified (UCSC Genome Browser on Human GRCh37/hg19 Assembly). The lower panel shows the key annotated genes.

to be due to oxygen deprivation. However, the identical twin sister of the patient who does not carry the same microduplication was physically and psychologically normal. Using recent SNP array technology, the patient was diagnosed with 1q21.1 duplication syndrome. To the best of our knowledge, the present study was the first to report a patient with 1q21.1 duplication syndrome in mainland China.

The chromosome 1q21.1 locus is a complex region with multiple low-copy repeats that make the region susceptible to recurrent deletions and duplications. Large rare copy number variants (CNVs) at this locus as well as microdeletions and microduplications have recently been associated with genomic disorders (OMIM nos. 612474 and 612475), characterized by developmental delay, neuropsychiatric abnormalities, dysmorphic features and a variety of congenital malformations $(1,2,13,14)$. Two main classes of 1q21.1 CNVs, spanning 20-40 genes, have been described. The more common one (class I; 1.8 Mb) includes only the distal 1q21.1 region, whereas the longer one (class II, $\sim 2.7 \mathrm{Mb}$ ) extends proximally to encompass the thrombocytopenia absent radius (TAR) syndrome region (2). Congenital heart defect is a major feature of 1q21.1 deletion $(3,5,6)$, and has been occasionally reported in association with 1q21.1 duplication $(8,15)$. The prevalence of CHD in a reported series of 1q21.1 deletions was $\sim 30 \%$ (3). The anatomic types were heterogeneous, mainly comprising left-sided obstructions (40\%), including aortic coarctation, bicuspid aortic valve and subaortic stenosis, but also septal defects (27\%) and conotruncal anomalies (20\%) (1). Of note, 1q21.1 duplication was more common in patients with tetralogy of fallot (8).

In the 1q21.1 locus, the genes GJA5 (7), CHD1 L (8) and PRKAB2 (16) were reported to be closely associated with CHD. The observation that none of them were included in the base sequence of the proband inferred that there may be other genes responsible for the occurrence of CHD. In the present study, the identified chromosome region contained $~ 30$ annotated genes, including HFE2, HYDIN2, RBM8A and CD160. To date, only few studies have associated duplications of these genes with CHD (17,18); the findings of the present study led to the hypothesis that the duplication of these genes caused an increased expression of the coded proteins, which in turn led to CHD. HFE2, which is also in this locus, encodes hemojuvelin (HJV), a protein involved in the activation of hepcidin and iron metabolism. Primarily, HJV is expressed in skeletal muscles, but it is also present at lower levels in the heart and the liver (17). Furthermore, a soluble form of HJV circulates in plasma. Mutations of this gene are known to be associated with hereditary juvenile hemochromatosis $(17,18)$. The gene HYDIN2, a paralogous segment of the primary ciliary dyskinesia-associated gene HYDIN (19), is involved in the development and formation of organ barriers, and its abnormity also causes congenital heart defects. Deletion/mutation of RBM8A can cause TAR syndrome, characterized by a series of phenotypes including coarctation of the aorta, left ventricular hypertrophy and sub-endocardial fibrosis (20); however, it has remained elusive whether duplication of this gene results in congenital heart defects. To confirm this hypothesis, further clinical and molecular biological studies are required.

Given that 1q21.1 duplication may not be well known to clinical cardiologists, the present study will facilitate the clinical recognition of 1q21.1 duplication in China. The results of the high-resolution SNP array in combination with the detailed phenotype analysis provided further evidence for the identification of causative genes for CHD in the 1q21.1 duplication. The present study may aid in the early diagnosis, 
genetic counseling and effective long-term management of 1q21.1 duplication.

\section{Acknowledgements}

The present study was supported by the National Natural Science Foundation of China (grant nos. 81200087, 81101475 and 81370204). The authors would like to thank the patient and her family members for participating in the present study. The authors would also like to thank the State Key Laboratory of Medical Genetics of China for their technical assistance.

\section{References}

1. Digilio MC, Bernardini L, Consoli F, Lepri FR, Giuffrida MG Baban A, Surace C, Ferese R, Angioni A, Novelli A, et al: Congenital heart defects in recurrent reciprocal 1q21.1 deletion and duplication syndromes: Rare association with pulmonary valve stenosis. Eur J Med Genet 56: 144-149, 2013.

2. Dolcetti A, Silversides CK, Marshall CR, Lionel AC Stavropoulos DJ, Scherer SW and Bassett AS: 1q21.1 Microduplication expression in adults. Genet Med 15: 282-289, 2013.

3. Mefford HC, Sharp AJ, Baker C, Itsara A, Jiang Z, Buysse K, Huang S, Maloney VK, Crolla JA, Baralle D, et al: Recurrent rearrangements of chromosome 1q21.1 and variable pediatric phenotypes. New Engl J Med 359: 1685-1699, 2008.

4. Brunet A, Armengol L, Heine D, Rosell J, García-Aragonés M, Gabau E, Estivill X and Guitart M: BAC array CGH in patients with Velocardiofacial syndrome-like features reveals genomic aberrations on chromosome region 1q21.1. BMC Med Genet 10: 144, 2009.

5. Brunetti-Pierri N, Berg JS, Scaglia F, Belmont J, Bacino CA, Sahoo T, Lalani SR, Graham B, Lee B, Shinawi M, et al: Recurrent reciprocal 1q21.1 deletions and duplications associated with microcephaly or macrocephaly and developmental and behavioral abnormalities. Nat Genet 40: 1466-1471, 2008.

6. Christiansen J, Dyck JD, Elyas BG, Lilley M, Bamforth JS, Hicks M, Sprysak KA, Tomaszewski R, Haase SM, Vicen-Wyhony LM, et al: Chromosome 1q21.1 contiguous gene deletion is associated with congenital heart disease. Circ Res 94: $1429-1435,2004$.

7. Guida V, Ferese R, Rocchetti M, Bonetti M, Sarkozy A, Cecchetti S, Gelmetti V, Lepri F, Copetti M, Lamorte G, et al: A variant in the carboxyl-terminus of connexin 40 alters GAP junctions and increases risk for tetralogy of Fallot. Eur J Hum Genet 21: 69-75, 2013.
8. Soemedi R, Topf A, Wilson IJ, Darlay R, Rahman T, Glen E, Hall D, Huang N, Bentham J, Bhattacharya S, et al: Phenotype-specific effect of chromosome 1q21.1 rearrangements and GJA5 duplications in 2436 congenital heart disease patients and 6760 controls. Hum Mol Genet 21: 1513-1520, 2012.

9. Slavotinek AM: Novel microdeletion syndromes detected by chromosome microarrays. Hum Genet 124: 1-17, 2008.

10. Chen JL, Yang YF, Huang C, Wang J, Yang JF and Tan ZP: Clinical and molecular delineation of 16 p13.3 duplication in a patient with congenital heart defect and multiple congenital anomalies. Am J Med Genet A 158A: 685-688, 2012.

11. Huang C, Yang YF, Yin N, Chen JL, Wang J, Zhang H and Tan ZP: Congenital heart defect and mental retardation in a patient with a 13q33.1-34 deletion. Gene 498: 308-310, 2012.

12. Tan ZP, Huang $\mathrm{C}, \mathrm{Xu} \mathrm{ZB}$, Yang JF and Yang YF: Novel ZFPM2/FOG2 variants in patients with double outlet right ventricle. Clin Genet 82: 466-471, 2012.

13. Cooper GM, Coe BP, Girirajan S, Rosenfeld JA, Vu TH, Baker C, Williams C, Stalker H, Hamid R, Hannig V, et al: A copy number variation morbidity map of developmental delay. Nat Genet 43: 838-846, 2011.

14. Rosenfeld JA, Traylor RN, Schaefer GB, McPherson EW, Ballif BC, Klopocki E, Mundlos S, Shaffer LG, Aylsworth AS and 1q21.1 Study Group: Proximal microdeletions and microduplications of 1q21.1 contribute to variable abnormal phenotypes. Eur J Hum Genet 20: 754-761, 2012.

15. Greenway SC, Pereira AC, Lin JC, DePalma SR, Israel SJ, Mesquita SM, Ergul E, Conta JH, Korn JM, McCarroll SA: De novo copy number variants identify new genes and loci in isolated sporadic tetralogy of Fallot. Nat Genet 41: 931-995, 2009.

16. Oliveira SM,Ehtisham J, Redwood CS, Ostman-Smith I, Blair EM and Watkins H; Mutation analysis of AMP-activated protein kinase subunits in inherited cardiomyopathies: Implications for kinase function and disease pathogenesis. J Mol Cell Cardiol 35: $1251-1255,2003$

17. Ganz T and Nemeth E: Hepcidin and disorders of iron metabolism. Annu Rev Med 62: 347-360, 2011.

18. Papanikolaou G, Samuels ME, Ludwig EH, MacDonald ML, Franchini PL, Dubé MP, Andres L, MacFarlane J, Sakellaropoulos N, Politou M, et al: Mutations in HFE2 cause iron overload in chromosome 1q-linked juvenile hemochromatosis. Nat Genet 36: 77-82, 2004.

19. Olbrich H, Schmidts M, Werner C, Onoufriadis A, Loges NT, Raidt J, Banki NF, Shoemark A, Burgoyne T, Al Turki S, et al: Recessive HYDIN mutations cause primary ciliary dyskinesia without randomization of left-right body asymmetry. Am J Hum Genet 91: 672-684, 2012.

20. Menghsol SC, Harris RD and Ornvold K: Thrombocytopenia and absent radii, TAR syndrome: Report of cerebellar dysgenesis and newly identified cardiac and renal anomalies. Am J Med Genet A 123A: 193-196, 2003. 